\section{Presbyphagia and dysphagia in old age}

\author{
Stefano Cavallero, Ligia J. Dominguez, \\ Laura Vernuccio, Mario Barbagallo \\ Geriatric Unit, Postgraduate School of \\ Geriatrics, University of Palermo, \\ Palermo, Italy
}

\section{Abstract}

Although presbyphagia is a clinically relevant problem among elderly population, possibly leading to dysphagia in old age, it remains a still underestimated health condition. The present review analyzes swallowing related anatomical and functional changes during aging, both in healthy people and in those affected by dysphagia. First of all, dysphagia in old people must not be confused with presbyphagia. To distinguish these two different conditions, a correct definition of both should be considered. Subsequently, a comprehensive evaluation including instrumental analysis should be carried out. The aim of this narrative review is to analyze the current knowledge of this clinical condition and to provide the state of art to clinicians. A systematic PubMed research on dysphagia in the elderly was conducted and most relevant and most recent references were manually screened and selected. The aim of a correct diagnosis is to enable the choice of a correct intervention in order to prevent and treat complications of dysphagia, such as $a b$ ingestis pneumonia and malnutrition. Moreover, the assessment, diagnosis and therapy of dysphagia/presbyphagia should include the intervention of different specialists.

\section{Introduction}

Swallowing is the act of bringing food, medications and saliva from the mouth to the stomach. Traditionally, it has been divided into three neuroanatomical phases: oral, pharyngeal, and esophageal. ${ }^{1}$ The complex of actions in swallowing involves a number of anatomical structures: muscles, nerves, teeth, receptors, salivary glands, soft and hard palate, true and false vocal folds, larynx, and hyoid bone. Nervous government of the swallowing is determined not only by brainstem but also by cerebral cortex: bilateral cortical input maintains facial tone and prevents spillage of oral content, and modulate patterned responses to govern mastication and bolus control during the oral phase. ${ }^{2}$ Precentral and inferior frontal gyrus are involved in facial, tongue, and masticatory coordination as well as pharyngeal phase activities such as hyoid elevation and vocal fold closure. ${ }^{3}$ Other regions adjacent to the Sylvian fissure and lateral precentral cortex have been shown to evoke swallowing responses associated with orofacial movements and salivation in humans $^{4}$ (Table 1). The aim of this narrative review is to analyze the current knowledge of this clinical condition and to provide the state of art to clinicians.

\section{Changes in swallowing in old age}

Aging is associated with multifactorial changes of swallowing physiology for which the term presbyphagia has been coined. ${ }^{5}$ Doty and Bosma ${ }^{6}$ were among the first to note swallowing changes in old age when they studied electromyographic responses during deglutition in young and old patients. They proposed that swallowing, like locomotion, was subject to different levels of arousal and pharyngeal stimulation, which diminishes as the person ages. Overall, few clinically significant abnormalities arise during the oral phase, and feeding performance does not significantly worsen with age in otherwise healthy patients. ${ }^{7}$ These are the result of generalized age-related changes in skeletal muscle strength, namely, sarcopenia: increased connective tissue within the body of the tongue restricts bolus control, requiring multiple tongue movements that hold the bolus more posteriorly and allow the bolus to enter the vallecula prematurely. ${ }^{8}$ These changes lead to a diminished lingual pressure reserve necessary to drive pharyngeal swallowing. ${ }^{9}$ Among healthy communitydwelling older adults, a positive correlation between posterior tongue strength (swallowing strength) and hand grip strength (muscle strength) was observed. ${ }^{10}$ Although feeding performance does not seem to be significantly affected by changes in oromotor skills, ${ }^{7}$ oral-phase problems are common because difficulty ingesting, controlling, or delivering the bolus relative to swallowing initiation is noted in $63 \%$ of healthy older adults. ${ }^{11}$ Like other functions in old age, we may call it homeostenosis in swallowing. Particularly, pharyngeal-phase abnormalities reduce the swallowing reserve, a measure of strength and coordination in excess of that needed to prevent aspiration. Manometric studies have demonstrated that the amplitude of pharyngeal pressures, duration of pressures, and rate of propagation are preserved in older patients. ${ }^{12}$ Despite the preservation of mus-
Correspondence: Stefano Cavallero, Geriatric Unit, Postgraduate School of Geriatrics, University of Palermo, viale del Vespro 141, 90127 Palermo, Italy.

Tel.: +39.091.6554813.

Fax: +39.091 .6552952 .

E-mail: stefano.cavallero@you.unipa.it

Key words: Dysphagia; presbyphagia; aging; malnutrition; tube feeding.

Contributions: all authors contributed substantially to the intellectual content, literature search and reviewing, writing and critically reviewing the manuscript.

Conflict of interests: the authors declare no potential conflict of interests.

Received for publication: 26 May 2020

Revision received: 3 July 2020.

Accepted for publication: 7 July 2020.

This work is licensed under a Creative Commons Attribution-NonCommercial 4.0 International License (CC BY-NC 4.0).

${ }^{\circ}$ Copyright: the Author(s), 2020

Licensee PAGEPress, Italy

Geriatric Care 2020; 6:9137

doi:10.4081/gc.2020.9137

cular activity, pharyngeal swallowing is delayed in healthy older adults relative to younger persons ${ }^{13}$ and they frequently require multiple swallows to effectively clear a bolus from the pharynx. For these reasons, older adults are three times more likely to have inspiration rather than expiration after swallowing and have more laryngeal penetration as evidenced by coughing and polyphasic laryngeal movements. ${ }^{14}$ Coupled with deficits in pharyngolaryngeal sensory discrimination that occur with age, ${ }^{15,16}$ this reduction in pharyngeal reserve may lead to silent aspiration in the otherwise healthy person, as demonstrated by Smith et al. in patients older than 90 years of age. ${ }^{17}$ There are contradictory studies regarding normal changes in upper esophageal sphincter (UES) function as we age. Although Ekberg and Feinberg ${ }^{11}$ reported defective opening of the cricopharyngeus muscle in $39 \%$ of elderly patients, subsequent manometric studies have demonstrated either no change or decreased resting tone in the UES of elderly patients. ${ }^{18}$ Delayed egress of material from the pharynx may still occur, despite normal cricopharyngeus muscle tone, as a result of increased connective tissue in the UES and decreased cross-sectional area of the esophageal inlet. ${ }^{1}$

In many studies, age is the single variable significantly associated with risk for 
dysphagia. ${ }^{19}$ Older people have functional changes inherent to aging itself and, more frequently, diseases that increase the swallowing disorder risk, ${ }^{20}$ such as Parkinson's disease, stroke, Alzheimer's disease, amyotrophic lateral sclerosis, head and neck cancer, and dementia. In addition, these individuals also present functional changes related with aging such as loss of strength and muscle tone, reduction of speed, precision and coordination of movements, reduction of propulsion and esophagi peristaltic reflexes, and tooth loss. ${ }^{21}$

\section{Dysphagia}

Dysphagia is a subjective sensation of difficulty or abnormality of swallowing. Six to ten million Americans currently report some degree of swallowing difficulty. ${ }^{22}$ Its prevalence in the general population ranges from $2 \%$ to $16 \%$, values that can be higher than $40 \%$ in hospitalized patients. ${ }^{5}$ Dysphagia is currently still associated with decreased quality of life, aspiration pneumonia, dehydration, malnutrition and social isolation. ${ }^{23}$ In hospital settings, dysphagia is also associated with longer length of hospital stay, higher costs, and higher mortality risk. ${ }^{24}$ Dysphagia is common among older people and it is associated with malnutrition, sarcopenia, dehydration, aspiration pneumonia, choking, and reduced ability to perform activities of daily living (ADL). Several factors explain the occurrence of poor muscle strength in older adults and how they may create a vicious cycle resulting in malnutrition. ${ }^{25}$ Reduced physical activity and functional decline, commonly observed in older adults, contribute to muscle atrophy and poor muscle strength. ${ }^{26,27}$ In addition, malnutrition promote body weight loss, particularly muscle mass loss and performance, including the deglutitive muscles, which consequently increasing swallowing problems and hence malnutrition risk. ${ }^{28,29}$ The prevalence of dysphagia in community-dwelling older people ( $>60$ years) ranged from $5 \%$ to $72 \%$ according to one systematic review, ${ }^{30}$ whereas another review reported that frequency of dysphagia in older people receiving long-term care ranged from $7 \%$ to $40 \%{ }^{31}$ Dysphagia is associated with malnutrition and reduced ADL in older persons receiving long-term care. ${ }^{32,33}$ Pneumonia, mainly aspiration pneumonia, is a common cause of death among older people. ${ }^{34}$

There are some different definitions of dysphagia based on both anatomical and functional problems. A traditional classification divided dysphagia in oropharyngeal dysphagia, characterized by difficulty initiating a swallow, and esophageal dysphagia, characterized by difficulty swallowing several seconds after initiating a swallow and a sensation of food getting stuck. ${ }^{35}$ Sarcopenic dysphagia is characterized by a decrease in the mass and loss of function in both whole body and swallowing muscles and is common in older people with sarcopenia and aspiration pneumonia. ${ }^{36,37}$

\section{Diagnosis}

Despite a greater understanding of swallowing physiology and advances in dysphagia evaluation, disorders of swallowing and feeding remain underappreciated by both the general public and physicians. ${ }^{38}$ Depression, cognitive dysfunction, and behavioral changes may delay the recognition of dysphagia in older adult patients by their providers. In addition, swallowing disorders are often insidious in their onset and may not manifest clinically for years to decades. Most abnormalities frequently lie clinically silent because the patient effectively compensates by changing diet consistency and feeding duration. ${ }^{1}$ In clinical practices we need simple, inexpensive, secure and fast tests to diagnose dysphagia. Evaluation of swallowing disorders in elderly patients begins with a comprehensive history and physical examination: careful questioning regarding eating habits, duration of feeding, diet, frequency of meals, and weight changes is essential. ${ }^{1}$ Clinical guidelines recommend early identification of dysphagia risk and, in this sense, the instrument for its screening represents a practical alternative of low cost that enable to identify early cases in which a detailed evaluation is required. ${ }^{39}$ Eating Assessment Tool (EAT-10) is a screening tool developed in the United States in 2008 using information collected from 482 patients. The EAT10 is considered a valid and solid selfassessment tool to measure dysphagia risk and identifies individuals that need early multidisciplinary intervention ${ }^{40}$ (Figure 1). The instrument has ten simple questions and provides information of functionality,

\begin{tabular}{|l|c|c|c|c|c|}
\hline Circle the appropriate response & \multicolumn{6}{|l|}{$0=$ No problem 4 = Severe problem } \\
\hline $\begin{array}{l}\text { 1. My swallowing problem has caused me to lose } \\
\text { weight. }\end{array}$ & 0 & 1 & 2 & 3 & 4 \\
\hline $\begin{array}{l}\text { 2. My swallowing problem interferes with my } \\
\text { ability to go out for meals. }\end{array}$ & 0 & 1 & 2 & 3 & 4 \\
\hline 3. Swallowing liquids takes extra effort. & 0 & 1 & 2 & 3 & 4 \\
\hline 4. Swallowing solids takes extra effort. & 0 & 1 & 2 & 3 & 4 \\
\hline 5. Swallowing pills takes extra effort. & 0 & 1 & 2 & 3 & 4 \\
\hline 6. Swallowing is painful. & 0 & 1 & 2 & 3 & 4 \\
\hline $\begin{array}{l}\text { 7. The pleasure of eating is affected by my } \\
\text { swallowing. }\end{array}$ & 0 & 1 & 2 & 3 & 4 \\
\hline 8. When I swallow food sticks in my throat. & 0 & 1 & 2 & 3 & 4 \\
\hline 9. I cough when I eat. & 0 & 1 & 2 & 3 & 4 \\
\hline 10. Swallowing is stressful & 0 & 1 & 2 & 3 & 4 \\
\hline Total EAT-10: & & & & & \\
\hline
\end{tabular}

Figure 1. EAT-10. Adapted from: Belafsky et al., 2008..$^{40}$

Table 1. Cerebral areas involved in swallowing.

Bilateral cortex

Precentral and inferior frontal gyrus

Regions adjacent to the Sylvian fissure and lateral precentral cortex
Maintains facial tone and prevents spillage of oral content, modulate patterned responses to govern mastication and bolus control during the oral phase

Involved in facial, tongue, and masticatory coordination, pharyngeal phase activities such as hyoid elevation and vocal fold closure

Evoke swallowing responses associated with orofacial movements and salivation 
emotional impact and physical symptoms that a swallowing problem can bring to an individual's life. ${ }^{41}$ Deglutition challenge at the bedside is actually debated, studies have demonstrated that bedside swallowing assessment fails to identify $33 \%$ to $50 \%$ of patients with significant aspiration on videofluoroscopy. ${ }^{42-44}$ Numerous noninvasive procedures, including respiratory pattern monitoring, pulse oximetry, cough reflex, ultrasonography, and acoustic monitoring, have been developed to assist the physician in identifying patients with swallowing inconpetence. ${ }^{45}$ Whereas dysphonia has been associated with aspiration following acute stroke, ${ }^{46}$ wet vocal quality alone failed to demonstrate significant association with aspiration on videofluoroscopy. ${ }^{45}$ Therefore, the first step in the systematic evaluation of dysphagia is a clinical swallowing evaluation. Patients that show any sign of dysphagia are referred for instrumental assessment if their condition allows it and if there is potential for change in the clinical management of the patient. ${ }^{47}$ The simplest evaluation of upper airway anatomy is barium swallow. During this study the patient swallows a suspension of barium with concurrent radiography of the neck and thorax. By varying the consistency of the barium suspension, one can evaluate swallowing competence and identify aspiration through laryngeal penetration of contrast agent. The addition of air-contrast granules may be used to distend the pharyngeal and esophageal lumen, providing visualization of subtle mucosal and submucosal lesions. ${ }^{1}$ The most important modification of the original barium swallow is the addition of videofluorography. Defined as the gold standard for swallowing evaluation and identification of aspiration, the videofluorography swallowing study (VFSS) provides a dynamic view of deglutition from the oral cavity to the lower esophageal sphincter and substantially extends the diagnostic capability of the study. ${ }^{1}$ Together with the VFSS, fiberoptic endoscopic evaluation of swallowing (FEES) is today the most commonly chosen method of swallowing assessment. ${ }^{48}$ In terms of day-to-day practice, the merits of FEES are that it can be performed at the bedside, thus facilitating examination of severely motorimpaired, bedridden or uncooperative patients; follow up examinations can be performed at short notice and, if necessary, frequently, and oropharyngeal secretion management and efficacy of cleaning mechanism, such as coughing and throat clearing, can be assessed simply and directly. Despite the numerous possible applications of FEES and undisputed need for qualified dysphagia assessment in this area of expertise, this technique is rarely taught systematically. ${ }^{47}$ Main issues against this technique are that it needs trained operators and the high cost of the device. Other swallowing studies are available to address specific abnormalities noted by radiographic imaging. Abnormalities in the region of UES or lower esophageal sphincter (LES) may be studied using manometry. ${ }^{49}$ Electromyography may also be useful in delineating the functional UES and has been described in the performance of percutaneous chemical myotomy using botulinum toxin. ${ }^{50}$

\section{Clinical management of dysphagia}

Dysphagia management is an important current and future public health issue in a society in which the average life expectancy is increasing. People with severe dysphagia who receive nil by mouth have a lower quality of life. ${ }^{36}$ The first approach in older persons is to avoid as many as possible con- ditions that may lead to dysphagia. The number of both prescription and nonprescription medications that have the undesirable effects on swallowing is large and spans several classes of pharmacological agents (Table 2). The most common cause of medication-induced dysphagia is xerostomia. Because saliva production is controlled by parasympathetic stimulation of muscarinic acetylcholine receptors, xerostomia results from all medications with significant anticholinergic activity. These effects are dose-dependent and may worsen with age despite stable dosing, because of age-related changes in drug metabolism and clearance. ${ }^{51}$ Common classes of drugs with anticholinergic side effects are antihistamines, tricyclic antidepressants, neuroleptics, antiemetics, atropine-containing antidiarrheal agents and anti-Parkinson medications. ${ }^{52}$ In addition to direct cholinergic interference on saliva production, diuretics frequently worsen xerostomia indirectly through dehydration. Many medications that alter or depress central nervous system activity may cause dysphagia in older adults, because of maintaining an individual's level of arousal is important to the muscular tone and coordination, which includes swallowing ability. Anxiolytics such as benzodiazepines and alcohol may be associated with dysphagia. Antihistamines, phenothiazine-based antiemetics, anticonvulsants, antipsychotics, opiates, and lithium also impair cognitive function and level of alertness. Treatment of schizophrenia in the geriatric population presents additional difficulties with swallowing dysfunction. In addition to variable anticholinergic and central nervous system depressant activity, neuroleptic medications may cause extrapyramidal symptoms manifesting as hyperfunctional involuntary movements of the oral or pharyngeal musculature. ${ }^{53}$ Rigidity and spasm

Table 2. Drugs associated with dysphagia in old people.

\begin{tabular}{ll}
\hline Medication altering cognitive function/alertness & - Antiepileptics \\
& - Antianxiety \\
& - Antihypertensives, especially centrally acting \\
& - Alcohol \\
Medications producing xerostomia & - Anticholinegics (e.g., sedating antihistamines, medication used for Parkinson's Disease) \\
& - Antipsychotics \\
& - Antidepressants \\
& - Retinoides \\
& - Antibiotics \\
& - Steroids \\
- Nonsteroidal anti-inflammatory drugs \\
- Biphosphonates \\
- Other (e.g., warfarin, diazepam, phenobarbital)
\end{tabular}


of the pharyngeal musculature may also result because these neuroleptics block dopamine receptors and may induce a druginduced Parkinsonism in $12 \%$ to $45 \%$ of patients. ${ }^{54}$ Such side effects are more common in older adults ${ }^{55}$ and may lead to aspiration through bolus misdirection during oral and pharyngeal transport. Therefore, also in dysphagia, wise deprescription is often the right solution. ${ }^{56,57}$

Poor oral hygiene is a risk factor for pneumonia, and aspiration of saliva, whether or not it is combined with food or fluid, can increase the likelihood of infection. Therefore, patients should be encouraged to perform oral hygiene several times a day and undergo periodic dental examinations. Furthermore, products to relieve oral dryness, as well as alcohol free mouth care products, can be recommended. Treatment for senescent dysphagia is usually compensatory, rehabilitative, or a combination of the two approaches. Compensatory interventions avoid or reduce the effects of impaired structures or neuropathology and resultant disordered physiology and biomechanics on bolus flow. Rehabilitative interventions have the capacity to directly improve dysphagia at the biological level. ${ }^{58}$ Postural adjustments are relatively simple to teach to a patient, require little effort to employ, and can eliminate misdirection of bolus flow through biomechanical adjustment. A general postural rule for facilitating safe swallowing is to eat in an upright posture $\left(90^{\circ}\right.$ seated) so that the vertical phases (pharyngeal) of the oropharyngeal swallow as well as esophageal motility capitalize on gravitational forces (Figure 2). Upright posture also can assist in precluding early spillage of food or liquid from horizontal oral phase into the regurgitation. A less obvious postural adjustment is useful for patients with hemiparesis. For this group of patients, a common strategy is a head turn toward the hemiparetic side, effectively closing off that side to bolus entry and facilitating bolus transit through the non-paretic pharyngeal channel. If the pathophysiologic condition is the uncoupling of the oral from the pharyngeal phase of the swallow indicated by a delay in onset of airway protection, a simple chin tuck $\left(45^{\circ}\right)$ reduces the speed of bolus passage, thereby giving the neural system the time it needs to initiate the pharyngeal and airway protection events prior to bolus entry. Eating an adequate amount of food becomes a challenge not only because of the increased time required to do so but also because fatigue frequently becomes an issue. To promote a safe, efficient swallow in most individuals with swallowing and chewing difficulties, some recommendations are useful: eat slowly with intent to implement control of bolus flow and allow enough time for a meal, do not eat or drink when rushed or tired, take small amounts of food or liquid into the mouth (use a teaspoon rather than a tablespoon), concentrate on swallowing only (eliminate distractions like television), avoid mixing food and liquid in the same mouthful (single textures are easier to swallow than multiple textures), avoid powders and dry foods in order to avoid small particles from entering the airway. The most common compensatory intervention is diet modification, a totally passive environmental adaptation. Withholding thin liquids such as water, tea, or coffee, which are most easily aspirated by older adults, and restricting liquid intake to thickened liquids is almost routine in nursing homes in an attempt to minimize or eliminate thin-liquid aspiration, presumably the precedent to the long-term related outcome, which is pneumonia. Increasing the viscosity of liquids using thickener additives decreases the rate of flow allowing patients more time to initiate airway protection and help to prevent or decrease the risk of aspiration. Despite the relevant impact these seemingly unappealing practices may have on the patient's quality of life, they have been commonly implemented in the absence of efficacy data. The American Dietetics Association (ADA)
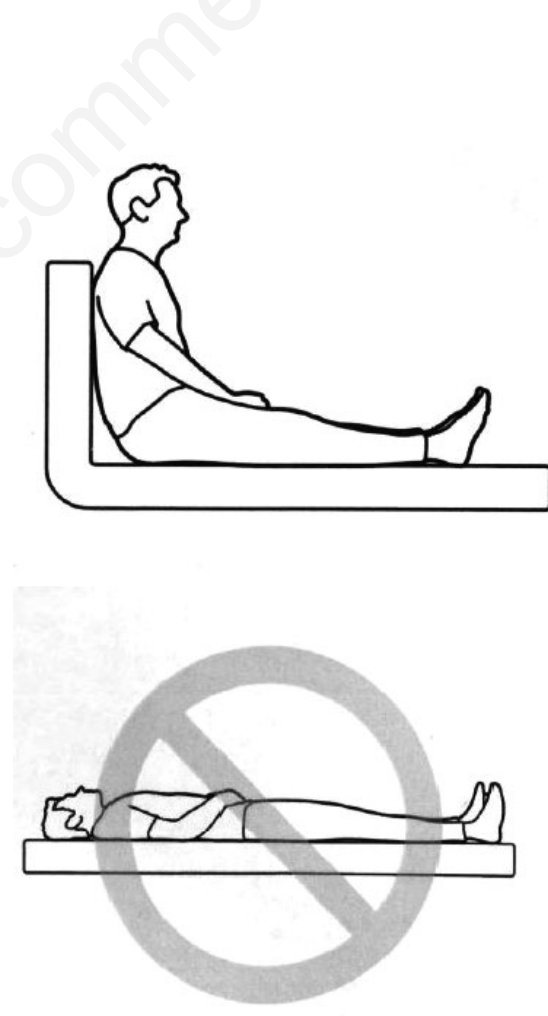

Figure 2. Correct position during the meal and positions to avoid in order to prevent food penetration and aspiration.

proposes to standardize dysphagia diets by modifications in the texture of solid foods and/or increased viscosity of liquids ${ }^{59}$ (Table 3). The ADA divided the texture of the food into 4 levels. Level 1 or the pureed diet includes cohesive foods that do not require bolus formation, controlled manipulation or chewing; it is indicated for individuals who have moderate to severe dysphagia and reduced ability to protect their airway. Level 2 or the mechanically altered diet includes foods that are moist, soft-textured, and easily formed into a bolus; this diet is a transition from the pureed textures to more solid textures and chewing ability is required. Level 3 or dysphagia advanced includes moist foods of nearly regular textures with the exception of hard and sticky foods; this diet is a transition to a regular diet and is appropriate for individuals with mild oral and/or pharyngeal phase dysphagia. ${ }^{60}$ Small frequent meals are often used for individuals with dysphagia, although, recent data suggests five daily feedings does not improve energy intake when compared with three feedings. ${ }^{61}$ In the end, the goal is to maintain an adequate nutrition and hydration, and the diet should be individualized, even with the help of the nutritionist. A better nutritional status correlated independently with an improved swallowing function. Maintaining an adequate nutritional status in patient at an acute hospital was reported to be associated with
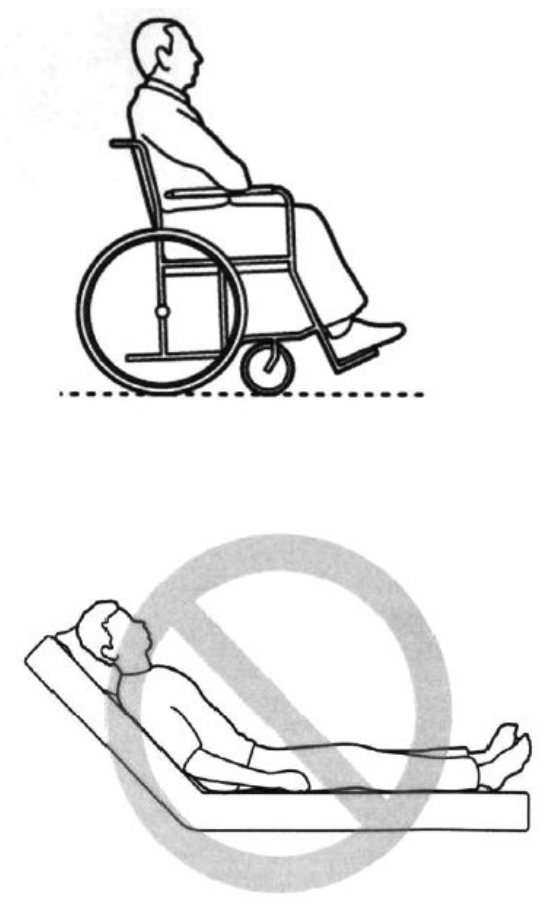
Table 3. National dysphagia diet progression.

\begin{tabular}{|c|c|c|}
\hline Level & Description & Rationale \\
\hline Level 1: dysphagia pureed & $\begin{array}{l}\text { This diet consists of pureed, homogenous, and cohesive } \\
\text { foods. Food should be pudding-like. } \\
\text { No coarse textures, raw fruits or vegetables, nuts, and so } \\
\text { forth allowed. } \\
\text { Any foods that require bolusformation, controlled } \\
\text { manipulation, or mastication are excluded }\end{array}$ & $\begin{array}{l}\text { This diet is designed for people who have moderate to severe } \\
\text { dysphagia, with poor oral phaseabilities and reduced ability to } \\
\text { protect their airway. Close or complete supervision and alternate } \\
\text { feeding methods may be required }\end{array}$ \\
\hline $\begin{array}{l}\text { Level 2: dysphagia } \\
\text { mechanically altered } \\
\text { (dysphagia ground) }\end{array}$ & $\begin{array}{l}\text { This level consists of foods that are moist, soft-textured, } \\
\text { and easily formed into a bolus. } \\
\text { Meats are ground or are minced no larger than } \\
\text { one-quarter-inch pieces; they are still moist, with some } \\
\text { cohesion. All foods from NDD Level } 1 \text { are acceptable at } \\
\text { this level }\end{array}$ & $\begin{array}{l}\text { This diet is a transition from the pureed textures to more solid } \\
\text { textures. Chewing ability is required. The textures on this level are } \\
\text { appropriate for individuals with mild to moderate oral } \\
\text { and/orpharyngeal dysphagia. Patients should be assessed for } \\
\text { tolerance to mixed textures. It is expected that some mixed } \\
\text { textures are tolerated on this diet }\end{array}$ \\
\hline Level 3: dysphagia advanced & $\begin{array}{l}\text { This level consists of food of nearly regular textures with } \\
\text { the exception of very hard, sticky, or crunchy foods. } \\
\text { Foods still need to be moist and should be in bite-size } \\
\text { pieces at the oral phase of the swallow }\end{array}$ & $\begin{array}{l}\text { This diet is a transition to a regular diet. Adequate dentition and } \\
\text { mastication are required. The textures of this diet are appropriate } \\
\text { for individuals with mild oral and/or pharyngeal phase dysphagia. } \\
\text { Patients should be assessed for tolerance of mixed textures. It is } \\
\text { expected that mixed textures are tolerated on this diet }\end{array}$ \\
\hline
\end{tabular}

Level 4: normal All foods

Modified from National Dysphagia Diet Task Force, 2002.59

better outcomes for dysphagia rehabilitation. ${ }^{62,63}$ Rehabilitative exercises are, by nature, more active and rigorous than alternative interventions for dysphagia. Research on the benefits of lingual resistance exercise suggests that strength-building exercises for the tongue increase lingual muscle strength and mass and improve the timing of the swallowing components in healthy older adults, with implications for greater gains and carryover into swallowing-related outcomes in elderly dysphagic patients..$^{9,64}$ Many studies describe benefits with isotonic and isometric resistance neck ${ }^{65,66}$ and tongue ${ }^{67}$ exercises, involving muscles that participate to the deglutition, such as mylohyoid and geniohyoid muscle groups, digastric muscle, tongue and the whole oropharyngeal musculature. Such findings suggest that older, dysphagic individuals are able to benefit from rehabilitative exercises focused on bulbar-innervated head and neck musculature. Dedicated and trained professional people, logopedists, should run these exercises in order to have the maximum benefit.

\section{Tube feeding}

Oropharyngeal dysphagia is potentially life threatening. In older population, crucial decisions often must be taken that influence the patient's safety, health and quality of life. Among these perplexing issues is the question of continuing oral intake or providing non-oral enteral nutrition. Percutaneous endoscopic gastrostomy
(PEG) tubes were first introduced into practice in 1980 with the purpose of providing enteral nutrition in children and young adults. ${ }^{68}$ They are currently the preferred method of delivering long-term nutrition when oral intake is deemed inadequate; PEG tube placement rates have risen steadly over the last twenty years from 15.000 in 1989 to 121.000 in 1995 and to more than 216.000 in the year $2000 .{ }^{69,70}$ While PEG tube placement is a relatively easy procedure with low procedure-related mortality (approximately $1 \%$ to $2 \%$ ), ${ }^{71,72}$ it is not without risks. Reviewing the literature provides a list of adverse events related to this procedure that can include local complications (such as wound infection, bleeding at the insertion site, leakage around the site, tube occlusion, erosion of the bumper into the abdominal wall, abdominal wall abscess, necrotizing fasciitis, colocutaneous fistula), gastrointestinal complications (ileus, diarrhea, nausea, vomiting, increased reflux), and other complications such as aspiration pneumonia, metabolic or electrolyte disturbance with refeeding, need for restraint use, loss of pleasure of eating, and loss of the social interaction with feeding. ${ }^{69,71,73,74}$ Among older adults requiring tube feeding, the most well studied subgroup are patients with dementia. Approximately $30 \%$ of all PEG tubes are placed in patients with dementia and as many as $10 \%$ of institutionalized older adults are being tube fed. ${ }^{75}$ Indeed, in the main studies evaluating survival in patients affected by dementia with or without PEG, there is no significant difference between the two groups. ${ }^{75}$ Furthermore, there are no evidences that it improves the quality of life. ${ }^{76}$

\section{Conclusions}

In conclusion, dysphagia is often an underestimated problem, expanding together with the aging of the population, and which is often diagnosed late, when the consequences can already be irremediable and fatal. The anatomical distinction between oropharyngeal and esophageal dysphagia, which can be useful from a surgical point of view, does not reflect the alterations that occur at the level of all stages of swallowing in neurogenic or functional dysphagia, in contrast with mechanical dysphagia, which often has reversible causes and can occur in younger patients. The strategies to be implemented cannot disregard a collegial management of the patients with dysphagia, where several health figures and different specialists collaborate, starting from primary care because the intervention is more effective the earlier the problem is diagnosed.

\section{References}

1. Schindler JS, Kelly JH. Swallowing disorders in the elderly. Laryngoscope 2002;112:589-602.

2. Plant RP. Anatomy and physiology of swallowing in adults and geriatrics. Otolaryngol Clin North Am 1998; 31:477-88.

3. Martin RE, Sessle BJ. The role of cerebral cortex in swallowing. Dysphagia 1993;8:195-202.

4. Miller AJ. The search for the central 
swallowing pathway: the quest for clarity. Dysphagia 1993;8:185-94.

5. Baijens LW, Clavé P, Walshe M, et al. European Society for Swallowing Disorders-European Union Geriatric Medicine Society white paper: oropharyngeal dysphagia as a geriatric syndrome. Clin Interv Aging 2016;11: 1403-28.

6. Doty RW, Bosma JF. An electromyographic analysis of reflex deglutition. J Neurophysiol 1956;19:44-60.

7. Fucile S, Wright PM, Gisel EG, et al. Functional oral-motor skills: do they change with age? Dysphagia 1998;13: 195-201.

8. Jaradeh S. Neurophysiology of swallowing in the aged. Dysphagia 1994;9: 218-20.

9. Nicosia MA, Hind JA, Robbins J, et al. Age effects on the temporal evolution of isometric and swallowing pressure. $\mathrm{J}$ Gerontol A Biol Sci Med Sci 2000;55: M634-40.

10. Butler SG, Stuart A, Kritchevsky SB, et al. The relationship of aspiration status with tongue and handgrip strength in healthy older adults. J Gerontol A Biol Sci Med Sci 2011;66:452-8.

11. Ekberg O, Feinberg MJ. Altered swallowing function in elderly patients without dysphagia. AJR Am J Roentgenol 1991;156:1181-4.

12. Robbins J, Hamilton JW, Lof GL, Kempster GB. Oropharyngeal swallowing in normal adults of different ages. Gastroenterology 1992;103:823-9.

13. Tracey JF, Logemann JA, Krugler C, et al. Preliminary observations on the effects of age on oropharyngeal deglutition. Dysphagia 1989;4:90-4.

14. Nilsson H, Ekberg O, Olsson R, Hindfelt B. Quantitative aspects of swallowing in an elderly nondysphagic population. Dysphagia 1996;11:180-4.

15. Calhoun KH, Gibson B, Hokanson JA, et al. Age-related changes in oral sensation. Laryngoscope 1992;102:109-16.

16. Aviv JE, Martin JH, Blitzer A, et al. Age-related changes in pharyngeal and supraglottic sensation. Ann Otol Rhinol Laryngol 1994;103:749-52.

17. Smith CH, Logemann JA, Pauloski BR, et al. Incidence and patient characteristics associated with silent aspiration in the acute care setting. Dysphagia 1999;14:1-7.

18. Shaker R, Ren J, Sui Z, et al. Effect of aging, position and temperature on the threshold volume triggering pharyngeal swallows. Gastoenterology 1994;103: 823-9.

19. Amaro PA, Araujo Dos Santos C, Firmino $\mathrm{HH}$, de Oliveira CBR. The importance of dysphagia screening and nutritional assessment in hospitalized patients. Einstein 2018;16:eAO4189.

20. Turley R, Cohen S. Impact of voice and swallowing problems in the elderly. Otolaryngol Head Neck Surg 2009;140: 33-6.

21. Eslick GD, Talley NJ. Dysphagia: epidemiology, risk factors and impact on quality of life - a population-based study. Aliment Pharmacol Ther 2008; 27:971-9.

22. Erlichman M. The role of speech-language pathologists in the management of dysphagia. Health Technol Assess Rep 1989;1:1-10.

23. Boccardi V, Ruggiero C, Patriti A, Marano L. Diagnostic assessment and management of dysphagia in patients with Alzheimer's disease. J Alzheimers Dis 2016;50:947-55.

24. Altman KW. Dysphagia evaluation and care in the hospital setting: the need for protocolization. Otolaryngol Head Neck Surg 2011;145:895-8.

25. Chatindiara I, Allen J, Wham C, et al. Dysphagia risk, low muscle strength and poor cognition predict malnutrition risk in older adults at hospital admission. BMC Geriatr 2018; 18:78.

26. Baumgartner RN, Waters DL, Garry PJ, et al. Predictors of skeletal muscle mass in elderly men and women. Mech Ageing Dev 1999;107:123-36.

27. Colón-Emeric CS, Withson HE, Pavon J, Hoenig H. Functional decline in older adults. Am Fam Physician 2013;88: 388-96.

28. Visvanathan R. Under-nutrition in older people: a serious and growing global problem! J Postgrad Med 2003; 49:352-60.

29. Lee JS, Frongillo Jr EA. Factors associated with food insecurity among US elderly persons importance of functional impairments. J Gerontol B Psychol Sci Soc Sci 2001;56:S94-9.

30. Madhavan A, LaGorio LA, Crary MA, et al. Prevalence of and risk factors for dysphagia in the community dwelling elderly: a systematic review. J Nutr Health Aging 2016;20:806-15.

31. Namasivayam AM, Steele CM. Malnutrition and dysphagia in longterm care: a systematic review. J Nutr Gerontol Geriatr 2015;34:1-21.

32. Wakabayashi H, Matsushima M. Dysphagia assessed by the 10-item Eating Assessment Tool is associated with nutritional status and activities of daily living in elderly individuals requiring long-term care. J Nutr Health Aging 2016;20:22-7.

33. Wakabayashi H, Matsushima M, Mutai
$\mathrm{R}$, et al. Occlusal support, dysphagia, malnutrition, and activities of daily living in aged individuals needing longterm care: a path analysis. J Nutr Health Aging 2018;22:53-8.

34. Maeda K, Akagi J. Muscle mass loss is a potential predictor of 90-day mortality in older adults with aspiration pneumonia. J Am Geriatr Soc 2017;65:e18-22.

35. Wakabayashi H, Matsushima M, Ichikawa $\mathrm{H}$, et al. The effects of resistance training of swallowing muscles on dysphagia in older people: a cluster, randomized, controlled trial. Nutrition 2018;48:111-6.

36. Wakabayashi H. Presbyphagia and sarcopenic dysphagia: association between aging, sarcopenia, and deglutition disorders. J Frailty Aging 2014;3:97-103.

37. Wakabayashi H, Sakuma K. Rehabilitation nutrition for sarcopenia with disability: a combination of both rehabilitation and nutrition care management. J Cachexia Sarcopenia Muscle 2014;5:269-77.

38. Gustafsson B, Tibbling L. Dysphagia: an unrecognized handicap. Dysphagia 1991;6:193-9.

39. Gonçalves MI, Remaili CB, Behlau M. Cross-cultural adaptation of the Brazilian version of the Eating Assessment Tool-EAT-10. Codas 2013; 25:601-4.

40. Belafsky PC, Mouadeb DA, Leonard AJ, et al. Validity and reliability of the Eating Assessment Tool (EAT-10). Ann Otol Rhinol Laryngol 2008;117:919-24.

41. Burgos R, Sarto B, Pérez-Portabella C, et al. Translation and validation of the Spanish version of the EAT-10 (Eating Assessment Tool-10) for the screening of dysphagia. Nutr Hosp 2012;27:2048-54.

42. Splaingard ML, Hutchins B, Sulton LD, Chaudhuri G. Aspiration in rehabilitation patients: videofluoroscopy versus bedside clinical assessment. Arch Phys Med Rehabil 1988;69:637-40.

43. Smithard DG, O'Neill PA, Morris J, et al. The natural history of dysphagia following a stroke. Dysphagia 1997;12: 188-93.

44. Martino R, Pron G, Diamant N. Screening for oropharyngeal dysphagia in stroke: insufficient evidence for guidelines. Dysphagia 2000;12:21-3.

45. Warms T, Richards J. "Wet voice" as a predictor of penetration and aspiration in oropharyngeal dysphagia. Dysphagia 2000;15:84-8.

46. Horner J, Massey EW, Brazer SR. Aspiration in bilateral stroke patients. Neurology 1990;40:1686-8.

47. Dziewas R, Baijens L, Clave P, et al. European society for swallowing disor- 
ders FEES accreditation program for neurogenic and geriatric oropharyngeal dysphagia. Dysphagia 2017;32:725-33.

48. Langmore SE. History of fiberoptic endoscopic evaluation of swallowing for evaluation and management of pharyngeal dysphagia: changes over the years. Dysphagia 2017;32:27-38.

49. Kelly JH. Use of manometry in the evaluation of dysphagia. Otolaryngol Head Neck Surg 1997;116:355-7.

50. Blitzer A, Brin MF. Use of botulinum toxin for the diagnosis and management of cricopharyngeal achalasia. Otolaryngol Head Neck Surg 1997;116: 328-9.

51. Katzung BG. Cholinoceptor-blocking drugs. In: Katzung BG, ed. Basic and Clinical Pharmacology, 6th ed. Norwalk, CT: Appleton and Lange; 1995. pp102-14.

52. Nastri M, Bartoli G, De Colle P. Impact of anticholinergic burden on cognitive impairment, disability and malnutrition: a cross-sectional study among hospitalized older patients. Geriatric Care 2019;5:8473.

53. Brandt N. Medications and dysphagia: how do they impact each other? Nutr Clin Pract 1999;14:S27-30.

54. Casey DE. Neuroleptic-induced acute extrapyramidal syndromes and tardive dyskinesia. Psychiatr Clin North Am 1993;16:589-610.

55. Sokoloff LG, Pavlakovic R. Neuroleptic-induced dysphagia. Dysphagia 1997;12:177-9.

56. Rossi PR, Hegarty SE, Miozzo S, et al. General practitioner attitudes and confidence to deprescribing for elderly patients. Geriatric Care 2020;6:8703.

57. Thomas RB, Jang SR, Lombardi M, Maio V. The prescription paradox: a guide to appropriate prescribing in the elderly. Geriatric Care 2019;5:8523. 58. Ney DM, Weiss JM, Kind AJH, Robbins JA. Senescent swallowing: impact, strategies and interventions. Nutr Clin Pract 2009;24:395-413.

59. National Dysphagia Diet Task Force. The National Dysphagia Diet: standardization for optimal care. American Dietetic Association; Chicago, IL: 2002.

60. Kind AJH, Smith MA, Finch MD, et al. Bouncing-back: rehospitalization in patients with complicated transitions in the first thirty days after hospital discharge for acute stroke. Home Health Care Serv Q 2007;26:37-55.

61. Taylor KA, Barr SI. Provision of small, frequent meals does not improve energy intake of elderly residents with dysphagia who live in an extended-care facility. J Am Diet Assoc 2006;106:1115-8.

62. Iwamoto M, Higashibeppu N, Arioka Y, Nakaya Y. Swallowing rehabilitation with nutrition therapy improves clinical outcome in patients with dysphagia at an acute care hospital. J Med Invest 2014;61:353-60.

63. Remelli F, Volpato S. Sarcopenia and malnutrition: impact on the outcome in hospitalized patients. Geriatric Care 2018;4:7723.

64. Robbins J, Levine R, Luschei E, et al. Age effects on lingual pressure generation as a risk factor for dysphagia. J Gerontol A Biol Sci Med Sci 1995;50:M257-62.

65. Shaker R, Kern M, Bonnevier J, et al. Augmentation of deglutitive upper esophageal sphincter opening in the elderly by exercise. Am J Physiol 1997;272:G1518-22.

66. Shaker R, Cook IJ, Dodds WJ, Hogan WJ. Pressure flow dynamics of the oral phase of swallowing. Dysphagia 1988;3:79-84.
67. Robbins J, Kays SA, Taylor AJ, et al. The effects of lingual exercise in stroke patients with dysphagia. Arch Phys Med Rehabil 2007;88:150-8.

68. Gauderer MW, Ponsky JL, Izant RJ Jr. Gastrostomy without laparotomy: a percutaneous endoscopic technique. J Pediatr Surg 1980;15:872-5.

69. Roche V. Percutaneous endoscopic gastrostomy. Clinical care of PEG tubes in older adults. Geriatrics 2003;58:22-9.

70. Garrow D, Pride P, Delegge M, et al. Feeding alternatives in patients with dementia: examining the evidence. Clin Gastroenterol Hepatol 2007;5:1372-8.

71. Skelly RH, Kupfer RM, Rawlings JK, et al. Percutaneous endoscopic gastrostomy (PEG): change in practice since 1988. Clin Nutr 2002;21:389-94.

72. Johnston SD, Tham TCK, Mason M. Death after PEG: results of the National Confidential Enquiry into Patients Outcome and Death. Gastrointest Endosc 2008;68:223-7.

73. Finucane TE, Christmas C, Travis K. Tube feeding in patients with advanced dementia: a review of the evidence. JAMA 1999;282:1365-70.

74. DeLegge MH, McClave SA, Ginsberg GG, et al. Ethical and medicolegal aspects of PEG-tube placement and provision of artificial nutritional therapy. Gastrointest Endosc 2005;62:952-9.

75. Cervo FA, Bryan L, Farber S. To PEG or not to PEG: a review of evidence for placing feeding tubes in advanced dementia and the decision-making process. Geriatrics 2006;61:30-5.

76. Veronese N, Minto S, Bonso O, Merlo A. Importance of quality of life in people with dementia treated with enteral nutrition: the role of the nurse. Geriatric Care 2019;5:8253. 